\title{
Effect of Occupational Exposure to Pesticides on Cardiovascular System among Farmers: A Comparative Study
}

\author{
Sunitha M. ${ }^{1}$, Jatin Bhoopala S. ${ }^{2}$, SureshY Bondade ${ }^{3}$ \\ ${ }^{1}$ Associate Professor, Physiology Department, ${ }^{2}$ MBBS Student, ${ }^{3}$ Associate Professor, \\ Physiology Department, JJM Medical College, Davangere, Karnataka, India
}

\begin{abstract}
Background: Agriculturea very complex occupation, apart from increasing productivity also brings huge loss to community in terms of its ill effects on human health,plant and animal life. Adverse effects on cardiovascular system is likely to occur due to aggressive use of agrochemicals such as pesticides.

Objectives: To Study Changes in ECG due to pesticide exposure among farmers and to Compare these ECG Changes in those farmers with that of normal non-exposed subjects.

Materials and Method: A 12 lead ECG was recorded in apparenty healthy male farmers exposed to pesticides and normal non exposed age matched male control subjects and analysed for parameters such as heart rate, PR interval, QTC interval, QRS axis, ST and T wave changes and compared.

Results: This study reports significantly increased heart rate, prolonged QTC interval and non-specific ST and $\mathrm{T}$ wave changes among pesticide exposed farmers compared to non-exposed control subjects.

Conclusion: The current study concludes that there is a need to detect these ECG changes as early as possible so that future risk of cardiovascular disease can be prevented by adapting precautionary measures during application of pesticides. Some form of continuous cardiac monitoring seems advisable. Farmers can be advised to quit toxic chemical fertilizers \& pesticides and opt Organic ones to be safe from the hazardous implications of exposure.
\end{abstract}

Keywords: ECG; Pesticides; Farmers; Cardiovascular disease.

\section{Introduction}

Agriculture is the backbone of the Indian economy. Agriculture plays a key role in the overall economic output of a country. Since in this modern era of agriculture, because of insufficient rains and due to parched irrigation, there is a persistent crop loss due to invasion by pests, diseases and weeds before the harvest. These losses affect the livelihood of a farmer and their

\section{Corresponding Author: \\ Dr. Sunitha M.}

Associate Professor, Physiology Department, JJM

Medical College, Davangere, Karnataka, India

e-mail: sunithamprof@gmail.com families. To get best crop yield there was introduction of chemical fertilizers \& pesticides. In making India a self-sufficient nation in food grain production day by day resulted in rampant usage of chemical fertilizers $\&$ pesticides bringing delineation of soil with toxic chemicals entering the food chain building-up toxicity with huge loss to community in terms of its ill effects on humans,plant and animal life due to aggressive use of agrochemicals $^{[1][2]}$.

Farmers, mostly being illiterate or ignorant, may not consider the precautions while using pesticides, might inhale, as well ingest or inculcate the toxic chemicals from them by one way or the other due to unhygienic conditions.

Chemicals like endosulfan, tetradifon, pyridaben, 
lead and many other organo-phosphates are present in pesticides $^{[3]}$.

Cardiovascular diseases are one of the leading causes of death worldwide ${ }^{[4]}$. Nearly $50 \%$ of world's labour force is into agricultural sector ${ }^{[5]}$. The presence of these toxicants in farmers has the potential to affect cardiovascular health as well as electrocardiogram ${ }^{[6]}$. the present study is designed to detect the ill effects of exposure to fertilizers and pesticides on cardiovascular function.

ECG Recording is a simple non-invasive technique. Hence, this study is taken up to detect the Electrocardiographic changes in Asymptomatic Toxicant-Exposed Farmers, to study prevalence of such abnormal ECG findings and thereby predict future cardiovascular complications as early as possible. Farmers can be advised to quit toxic chemical fertilizers $\&$ pesticides and opt Organic ones to be safe from the hazardous implications of exposure.

\section{Methodology}

This study was undertaken in 60 apparently healthy male farmers, aged between 20 to 40 years with history of working for at least 6-8 hours a day for twice a week for more than 10 years of exposure to pesticides in the farm. Sixty-two apparently healthy male, control subjects were selected randomly from general population. We approached Rural Farmers' associations and APMC Farmers' association for selection of study subjects. Subjects will be matched for age, height and weight. Subjects with History of smoking, Alchohol intake. Diabetes mellitus, Hypertension, Anemia, Abnormalities of Vertebral column and thoracic cage, Pulmonary tuberculosis, Bronchial asthma, Chronic bronchitis, other respiratory diseases, Cardiac diseases, Abdominal or chest surgery were excluded from the study.

After taking written consent and asking detailed history about pesticide exposure with series of questionneries like type of pesticides, mixing procedure, use of protective measures like gloves and masks, frequency of application etc Institutional Ethics Committee (IEC) Clearance was obtained before beginning the study.

A detailed assessment of individual subjects was done using pre-structured pro-forma. Data acquisition was done in the morning. A detailed physical examination of subjects was done followed by systemic examination and ECG recording. Physical examination of all the subjects included temperature, blood pressure, respiratory rate and pulse rate.

Detailed history was taken which included a comprehensive history on usage of agrochemicals

1. Type \& composition (mix or solitary; pyridaben chlorpyroslurealorganochloridelammonia and many other compounds)

2. Duration of usage-daily \weekly \quantity \mixture

3. Precautions considered-masks $\backslash$ clothing $\backslash$ footwear $\backslash$ eye wearlpost-work sanitation

4. Type of dispersal-by-hand $\backslash$ sprayltablets

Resting pulse rate was recorded from the radial artery. Blood pressure was recorded using a mercury sphygmomanometer with the appropriately sized cuff from the right brachial artery in supine position.

ECG was recorded in those individuals who are selected for the study. The instrument used for recording ECG is Computerized NIVIQURE Digital 12 Lead ECG machine. Special emphasis was given on Heart Rate, PR Interval, QTc Interval, QRS axis, ST Segment, T Wave changes and all parameters will be analysed. ECG signals were obtained from all the leads. The following ECG parameters have been considered in this study.

Standard ECG reference values will be taken from Wagner G.S: Interpretation of normal electrocardiogram.

Statistical Analysis: Continuous variables will be expressed as mean \pm standard deviation (SD). Categorical data will be presented as frequencies and percentages. Differences between the groups will be compared using the two-tailed unpaired student's t-test. Comparisons of categorized variables between groups will be performed using the $\chi 2$ test. All tests of statistical significance will be two-sided and $\mathrm{P}<0.05$ will be considered to indicate a statistically significant difference. The statistical analyses will be conducted with SPSS 20.0

\section{Results}

The results obtained were expressed as mean \pm standard deviation. The study showed the following results which are tabulated and analysed. 
Table 1: Age-wise distribution of subjects in each group

\begin{tabular}{|l|c|c|c|}
\hline Age (Yrs.) & Farmers & Controls & Total \\
\hline $25-29$ & 20 & 26 & 46 \\
\hline $30-34$ & 16 & 26 & 42 \\
\hline $35-39$ & 23 & 31 & 54 \\
\hline $40-44$ & 32 & 15 & 47 \\
\hline 45 & 9 & 2 & 11 \\
\hline Total & 100 & 200 & \\
\hline Chi Square Test: $\mathrm{P}<0.000$ & & & \\
\hline
\end{tabular}

Table 2: Incidence Of occurrence of Heart Rate changes in farmers exposed and controls:

\begin{tabular}{|l|c|c|c|}
\hline HR (bpm) & Farmers & Controls & Total \\
\hline Normal & 88 & 98 & 186 \\
\hline Abnormal & 12 & 2 & 14 \\
\hline Total & 100 & 100 & 200 \\
\hline Chi Square Test: $P<0.01$, Significant & & \\
\hline
\end{tabular}

Table 3: Incidence of occurrence of changes in the ST segment in farmers exposed and controls:

A. With respect to the number of subjects showing the changes:

\begin{tabular}{|l|c|c|c|}
\hline ST Segment & Farmers & Controls & Total \\
\hline E & 3 & 0 & 3 \\
\hline IE & 97 & 100 & 197 \\
\hline Total & 100 & 100 & 200 \\
\hline Fisher's exact test: $\mathrm{P}<0.003$, Not Significant & \\
\hline
\end{tabular}

*where E-Elevation \& IE-Iso-Electric

B. With respect to the leads and amplitude:

\begin{tabular}{|c|c|c|c|}
\hline \multicolumn{3}{|c|}{ Farmers } \\
\hline Lead & No. Cases & Max (Amp) & No. Cases \\
\hline I & 1 & $0.5 \mathrm{~mm}$ & 1 \\
\hline II & 2 & $1 \mathrm{~mm}$ & \\
\hline III & 1 & & \\
\hline V3 & 2 & & \\
\hline V4 & 2 & & \\
\hline V5 & 1 & & \\
\hline
\end{tabular}

Table 4: Incidence of occurrence of changes in $T$ Wave in farmers exposed and controls

\begin{tabular}{|c|c|c|c|c|}
\hline T Wave & Farmers & Controls & Fisher's exact test & Significance \\
\hline I & 0 & 0 & - & - \\
\hline I & 0 & 0 & - & - \\
\hline III - I & 2 & 0 & $\mathrm{P}<0.311$ & NS \\
\hline III-F & 5 & 7 & & \\
\hline
\end{tabular}




\begin{tabular}{|c|c|c|c|c|}
\hline T Wave & Farmers & Controls & Fisher's exact test & Significance \\
\hline avR & 0 & 0 & - & - \\
\hline avL & 0 & 0 & - & - \\
\hline avF & 0 & 0 & - & - \\
\hline V1 & 3 & 0 & $\mathrm{P}, 0.123$ & NS \\
\hline $\mathrm{V} 2$ & 2 & 0 & $\mathrm{P}<0.249$ & NS \\
\hline V3 & 1 & 0 & $\mathrm{P}<0.500$ & NS \\
\hline V4 & 0 & 0 & - & - \\
\hline V5 & 0 & 0 & - & - \\
\hline V6 & 0 & 0 & - & - \\
\hline Total & 100 & 100 & - & - \\
\hline
\end{tabular}

Table 5: Comparison of QTc interval (seconds) in farmers exposed and controls

\begin{tabular}{|l|c|c|c|}
\hline QTc & Farmers & Controls & Total \\
\hline Normal & 83 & 98 & 181 \\
\hline Abnormal & 17 & 2 & 19 \\
\hline Total & $\mathbf{1 0 0}$ & $\mathbf{1 0 0}$ & $\mathbf{2 0 0}$ \\
\hline Chi Square Test P $<0.01$, Significant & & \\
\hline
\end{tabular}

Table 6: Comparison of mean values of clinical parameters in farmers exposed and controls: Heart rate (bpm), PR interval (sec), QTc interval (sec) and QRS axis (degrees):

\begin{tabular}{|c|c|c|c|c|c|c|}
\hline \multicolumn{2}{|c|}{ Groups } & $\mathbf{N}$ & Mean & Std. Deviation & P Value & Significance \\
\hline \multirow{2}{*}{ HR } & Farmers & 100 & 88.45 & 9.59 & \multirow{2}{*}{$\mathrm{p}<0.000$} & \multirow{2}{*}{ HS } \\
\hline & Controls & 100 & 82.70 & 9.80 & & \\
\hline \multirow{2}{*}{ PR } & Farmers & 100 & 0.14734 & 0.1123 & \multirow{2}{*}{$\mathrm{P}<0.870$} & \multirow{2}{*}{ NS } \\
\hline & Controls & 100 & 0.14762 & 0.128 & & \\
\hline \multirow{2}{*}{ QTc } & Farmers & 100 & 0.42241 & 0.142 & \multirow{2}{*}{$\mathrm{P}<0.000$} & \multirow{2}{*}{ HS } \\
\hline & Controls & 100 & 0.38860 & 0.298 & & \\
\hline \multirow{2}{*}{ QRS axis } & Farmers & 100 & 48.57 & 12.57 & \multirow{2}{*}{$\mathrm{P}<0.96$} & \multirow{2}{*}{ NS } \\
\hline & Controls & 100 & 48.66 & 12.89 & & \\
\hline
\end{tabular}

\section{Discussion}

Cardiovascular diseases (CVD) are associated with increased morbidity and mortality rates and reduced life expectancy ${ }^{[7]}$. Apart from the fundamental risk factors, environmental toxic substance including pesticides may affect cardiovascular system ${ }^{[8]}$. ECG is influenced by these changes due to the exposure to pesticides. A combination of these changes and continued exposure predisposes to various cardiovascular diseases which can turn out fatal. It is essential to understand the repercussions of these occupational exposures to pesticides and its associated future risk of cardiovascular diseases.

The current study showed the following results which is analysed as follows:

\section{ECG Report:}

Heart Rate: The incidence of occurrence of changes in Heart rate was significantly higher in the farmers exposed compared to the control group $(\mathrm{P}<0.000)$ (Table 2 ). There was a highly significant increase in the heart 
rate in exposed farmers group as compared to control to the control group $(\mathrm{P}<0.000)$

Our finding is in consistent with other studies.

Saadedeh AM and his coworkers in their study reported increase in heart rate compared to controls ${ }^{[9]}$.

Karki $\mathrm{P}$ and his colleagues found significant increase in heart rate as an electrocardiographical manifestation in pesticide exposed subjects ${ }^{[10]}$.

This increase in HR could be related to the nicotinic effects of OP compounds and other agrochemicals and increased sympathetic tone ${ }^{[11]}$.These compounds act by irreversibly inhibiting the enzyme cholinesterase, resulting in accumulation of acetylcholine at synapses and myoneural junctions leading to cholinergic overactivity ${ }^{[9][10]}$.

PR interval: In this study There was no significant change in the mean PR interval (seconds) in exposed farmers group as compared to the control group $(\mathrm{P}<0.870)$ (Table 3).

QTc interval: The mean QTc interval (seconds) in exposed farmers group was significantly prolonged compared to the control group $(\mathrm{P}<0.000)$ (Table 6) The QTc interval tended towards the upper end of the normal range.

Wahab A and his coworkers in their study showed increased QTc interval as an effect of pesticides on cardiovascular system ${ }^{[12]}$.

Mladenka $\mathrm{P}$ et al reported prolonged QTc interval in pesticide exposed subjects. Since QTc interval is more important parameter than QT interval since it is corrected with heart rate, here in this study special interest is given to QTc interval ${ }^{[13]}$.

The QT Interval is an element of ECG that is a representation of ventricular function which includes depolarization and re-polarization. Measurement of QT and QTc can be used as a simple indicator for cardiovascular diseases ${ }^{[14]}$.

In this study there was prolongation of QTc interval in fertilizer and pesticide-exposed farmers group which is highly significant compared to controls. The QTc interval tended towards the upper end of the range and significant number showed abnormal QTc prolongation. The QTc interval represents duration of activation of ventricular myocardium and its recovery. The QT interval corrected for heart rate (QTc) which is longer than 0.44 seconds is generally considered to be abnormal. The QT interval represents the duration of activation and recovery of the ventricular myocardium. Prolonged QTc interval here suggests prolonged recovery from electrical excitation which increases the likelihood of dispersing refractoriness, when some parts of myocardium might be refractory to subsequent depolarization ${ }^{[12]}$.

Prolonged QTc interval can be attributed to the due to direct cardiac toxicity (myocardial necrosis) along with the associated structural myocardial damage caused by pesticides ${ }^{[13]}$.

Studies done in animals reported that prolongation of the QT interval is a direct myocardial pesticide effect and is independent of cholinergic effects. ${ }^{\text {[9] }}$ The cardiovascular effects of organophosphorus compounds are unpredictable and often change and adverse effects increase with increase over the time course of exposure. ECG changes like QTc prolongation is potentially dangerous and indicates the requirements of continuous cardiac monitoring ${ }^{[11]}$.

ST segment: In this study non-specific ST segment elevation less than $1 \mathrm{~mm}$ was found out in three subjects in farmers exposed to fertilizers \& pesticides but it was found to be statistically not significant compared to the normal control groups.(Table 4)

These changes are significant seen in higher dose exposures and acute poisoning ${ }^{[13]}$.

T wave changes: In our studies, changes in $T$ wave were found to statistically not significant. But certain non-specific changes were seen in the farmers group such as inversions and flattened waves in a few leads which were low amplitude waves. (Table 5)

These non-specific changes in ST segment and $\mathrm{T}$ wave changes can be explained by the fact that the inorganic compounds in pesticides cause direct myocardiotoxic damage and studies have the revealed that patchy necrosis, cardiac discoloration, patchy pericarditis, auricular thrombus, right ventricular hypertrophy, myocardial interstitial oedema, vascular congestion, patchy interstitial inflammation and mural thrombus occurs in acutely exposed cases ${ }^{[13]}$.

QRS axis change: In the current study there was no significant difference in the mean QRS axis in 
exposed farmers group as compared to the control group $(\mathrm{P}<0.960)$ (Table 7)

\section{Conclusion}

The present study concluded thatOccupational exposure to pesticides is the major health concern of today's society, especially among farmers as they are mostly unaware of it and it can predispose them for the development of various morbid conditions over the years. The prevalence of unreported case of cardiovascular deaths is of serious concern. The number of farmers that use agrochemicals then retired due to ill health normally die without knowing the cause of their death. The banning of agrochemicals in the agricultural sectors will produce adverse economic effects and people will suffer across the world. As in low dose chronic exposure they may not manifest clinically, and pesticides exposed subjects for long time remain asymptomatic. However, it brings Electrocardiographic changes that are noticed early over a period of exposure and the general impression is that it is advisable to detect these changes as early as possible so that future risk of cardiovascular disease can be reduced by adapting precautionary measures during application of pesticides. Therefore, some form of continuous cardiac monitoring seems advisable. Considering the gravity of this situation, more work needs to be done in this aspect. Farmers can be advised to quit toxic chemical fertilizers \& pesticides and opt Organic ones to be safe from the hazardous implications of exposure.

Acknowledgement: Authors are grateful to Dr. S.B. Murugesh, Principal, JJM Medical College, Davangere for his support and encouragement. We also extend our thanks to our statistician. Authors are deeply indebted to all volunteers who participated in this study.

Conflict of Interest: None to declare.

Source of Funding: Self .

Ethical clearance from institution: Obtained

\section{References}

1. Sekhotha M, Monyeki K, Sibuyi M. Exposure to Agrochemicals and Cardiovascular Disease: A Review. International Journal of Environmental Research and Public Health. 2016 Feb 18; 13(2): 229.
2. Beyene N, Hans K, Yalemtshay M, Roel V. Use of Chemical Pesticides in Ethiopia: A Cross-Sectional Comparative Study on Knowledge, Attitude and Practice of Farmers and Farm Workers in Three Farming Systems. The Annuls of Occupational Hygiene. 2016 June 1;60(5):551-556.

3. Winand K, Eric SL. Toxicity of Pesticides. Pennsylvania State University. 2016.

4. Finegold, Judith A. et al. Mortality from ischaemic heart disease by country, region, and age: Statistics from World Health Organisation and United Nations International Journal of Cardiology. September 2013;168(2): 934 - 945.

5. Maroni M, Fanetti A, Metruccio F. Risk Assessment and Management of Occupational Exposure To Pesticides in Agriculture. University of Milan. 2006 April;97(2):430-7.

6. Ha MH, Lee DH, Jacobs DR. Association between serum concentrations of persistent organic pollutants and self-reported cardiovascular disease prevalence: results from the National Health and Nutrition Examination Survey, 1999-2002. Environ Health Perspect. 2007;115(8):1204-9.

7. Harpal S Buttar, Timao Li et al. Prevention of cardiovascular diseases: Role of exercise, dietary interventions, obesity and smoking cessation:Exp Clin Cardiol.2005; 10(4): 229-249.

8. Nicolopoulou-Stamati P, Maipas S, Kotampasi C, Stamatis P and Hens L: Chemical Pesticides and Human Health: The Urgent Need for a New Concept in Agriculture. Front. Public Health:2016. 4:148.

9. Saadeh AM, Farsakh NA, Al-Ali MK. Cardiac manifestations of acute carbamate and organophosphate poisoning. Heart. 1997;77(5):4614.

10. Karki P, Ansari JA, Bhandary S, Koirala S. Cardiac and electrocardiographical manifestations of acute organophosphate poisoning. Singapore Medic J. 2004; 45:385-9.

11. Tripathy S, Rout $P$ et at., Study of clinical profile of organophosphorus poisoning with special reference to electrocardiographic changes and electrolyte derangement. Int J Adv Med. 2018 Feb;5(1):50-56.

12. Wahab A, Hod R, Ismail NH, Omar N. The Effect of Pesticide Exposure on Cardiovascular System: A 
Systemic Review. International Journal Community Medicine \& Public Health. 2016;3: 1-10.

13. Mladěnka $P$, Applová L, Patočka J, et al. Comprehensive review of cardiovascular toxicity of drugs and related agents. Med Res Rev. 2018;38(4):1332-1403.
14. Pieter G. Postema, Arthur A.M. Wilde. The Measurement of the QT Interval Current Cardiology Reviews. 2014: 10, 287-294. 